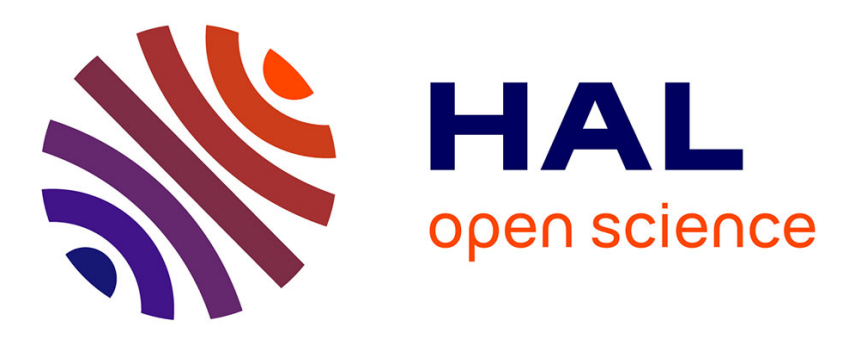

\title{
Sur la relation d'Élaboration: des approches intuitives aux approches formelles
}

\author{
Georges Kleiber, Hélène Vassiliadou
}

\section{To cite this version:}

Georges Kleiber, Hélène Vassiliadou. Sur la relation d'Élaboration: des approches intuitives aux approches formelles. Journal of French Language Studies, 2009, Relations de cohérence et fonctionnement des anaphores, 19 (special issue), pp.183-205. halshs-01076307

\section{HAL Id: halshs-01076307 https://shs.hal.science/halshs-01076307}

Submitted on 26 Oct 2014

HAL is a multi-disciplinary open access archive for the deposit and dissemination of scientific research documents, whether they are published or not. The documents may come from teaching and research institutions in France or abroad, or from public or private research centers.
L'archive ouverte pluridisciplinaire HAL, est destinée au dépôt et à la diffusion de documents scientifiques de niveau recherche, publiés ou non, émanant des établissements d'enseignement et de recherche français ou étrangers, des laboratoires publics ou privés. 


\title{
Sur la relation d'Élaboration: des approches intuitives aux approches formelles ${ }^{1}$
}

\author{
GEORGES KLEIBER ET HÉLÈNE VASSILIADOU \\ EA 1339-LILPA / Scolia et Université de Strasbourg
}

\section{INTRODUCTION}

Bien que l'existence de relations de cohérence ou de relations de discours ou encore relations rhétoriques soit admise communément, il n'y a pas de consensus ni quant à leur nombre - elles peuvent varier de deux (relations de dominance et de satisfaction-précédence chez Grosz et Sidner, 1986) à plus de cent (Martin, 1992) — ni quant à leur classification et dénominations $^{2}$ ni - ceci expliquant cela ou l'inverse - quant à leur définition. Ces divergences, souvent signalées dans la littérature, ne facilitent pas la tâche à ceux qui veulent les faire «tourner» sur les textes, car elles nécessitent un travail préalable de mise au point qui n'est pas toujours aisé à faire. C'est un tel travail que nous entreprendrons dans cet article: ${ }^{3}$ notre objectif est en effet d'examiner les tenants et aboutissants d'une des relations de discours les plus importantes, la relation dite d'Élaboration. La raison en est simple: si, intuitivement, elle paraît facile à saisir, elle est beaucoup plus délicate à définir avec précision et donne lieu à des interprétations qui ne sont pas toujours univoques.

Notre enquête partira des approches intuitives de la relation, puis, après avoir présenté la définition de la RST (Rhetorical Structure Theory) de Mann et Thompson (1987 et 1988), abordera successivement la définition «inférentielle » de Hobbs (1979 et 1990) et la conception de la SDRT (Segmented Discourse Representation Theory) dans la version d'Asher (1993) et celle d'Asher et Lascarides (1996 et 2003). Dans cette perspective, nous essaierons de mettre en relief les lignes de force et les tensions qui traversent les différentes conceptions examinées, avec comme objectif d'éclairer aussi bien les différentes théories ambiantes que la relation d'Élaboration elle-même. C'est ainsi que nous montrerons que les différentes définitions proposées sont, d'une part, travaillées par une tension entre l'identité et la différence et que, d'autre part, des approches formelles

\footnotetext{
1 Nous remercions F. Cornish et nos trois relecteurs anonymes pour leurs suggestions. Merci aussi à Laure Vieu de nous avoir fait part de son désaccord sur certaines de nos analyses, qui nous semblent malgré tout tenir la route.

${ }^{2}$ Pour les différentes oppositions, voir la vaste littérature consacrée au sujet : Sanders et al. 1992, 1993 ; Sanders 1997 ; Degand 1998 ; Knott et Sanders 1998 ; Oates 1999 ; Knott 2001 ; Knott et al. 2001 ; Rossari 2000 et 2001 ; Cornish 2006 a et b ; Taboada et Mann 2006, etc.

${ }^{3}$ Le présent article prolonge le travail commencé dans Kleiber et Vassiliadou (2007).
} 
comme celle d'Asher (1993) et Asher et Lascarides (2003) réunissent sous le même chapeau de l'Élaboration deux types de relation fort différentes, à savoir une relation de type hyperonymique (subsomption) et une relation de type "partie-tout".

\section{APPROCHES INTIUITIVES DE LA RELATION D'ÉLABORATION: « UN OU DES DETAILS ADDITIONNELS »}

En matière d'interprétation, c'est-à-dire en matière de sens, l'intuition ne peut être mise de côté. En matière de relations de cohérence, elle est tout particulièrement indiquée pour servir de guide, au moins comme point de départ. Pour la bonne et simple raison, que, intuitivement, nous reconnaissons des relations différentes entre les deux phrases des séquences (1), (2) et (3), respectivement celles de causalité, de succession et de conséquence, alors qu'aucune expression linguistique ne marque pourtant la relation établie :

(1) Paul est tombé. Pierre l'a poussé.

(2) Paul est tombé. Marie l'a relevé.

(3) Il a beaucoup plu. La rivière a débordé.

Cette reconnaissance repose sur des connaissances du monde (on sait qu'un surplus pluviométrique est susceptible d'engendrer des inondations), plus précisément sur le rapport que nous pouvons établir entre ce qui est dit dans la première phrase et ce qui est dit dans la seconde et qui assure la cohérence de l'ensemble: 'relations are suggested to make links between knowledge and render discourse coherent through their recognition' (Oates, 1999: 14). Les définitions plus précises, qu'elles soient formelles ou non, ne font qu'essayer de mettre en forme cette intuition (voir par exemple la formule suivante d'Asher et Lascarides, 2003: 16: 'assuming that its logical form is as intuition dictates...'). Il est donc tout à fait logique que nous commencions par une telle approche de la relation d'Élaboration en donnant à voir l'intuition qui est à son origine. Si l'on considère des exemples comme (4) et (5),

4) Il pleuvait. De grosses gouttes tombaient sur le sol.

5) Marie a travaillé toute la matinée. Elle a nettoyé toute la cuisine.

on voit intuitivement que la seconde phrase ne fait que préciser ou développer le contenu de la première en apportant une information supplémentaire, plus détaillée, plus précise sur elle. Dans l'exemple (4), la seconde phrase indique comment il pleut; en (5), elle précise quel a été le travail de Marie. Cette intuition, à savoir que la seconde phrase n'apporte qu'un « détail » additionnel à la première, est à l'origine de la dénomination 
anglaise Elaboration. ${ }^{4}$ Elle se retrouve chez tous ceux qui promeuvent la relation, comme le montrent les citations suivantes:

- Reader recognizes S as providing additional detail for N. Reader identifies the element of subject matter for which detail is provided ${ }^{5}$ (Mann et Taboada, 2005: 3); - [...] one clause expands another by elaborating on it (or some portion of it): restating in other words, specifying in greater detail, commenting, or exemplifying (Halliday, 1994: 220) ;

- (Elaboration) relates two propositions only if the second proposition provides more detail about the event described in the first. Introducing an Elaboration thus introduces a new level of detail into the discourse (Asher et Lascarides, 2003: 8).

L'impression qui se dégage des approches intuitives et des exemples sur lesquels elle a pris corps est celle d'un consensus sur le fait que, dans les cas typiques, il $y$ a addition informationnelle et que cette addition informationnelle est conçue comme étant mineure - l'essentiel de l'information ayant été donné par la première phrase - qu'il s'agit, en somme, de détails ou précisions ou spécifications sur la première phrase. Ceci signifie qu'avec la deuxième phrase on ne sort pas du cadre ou de la situation brossée ou encore du topique ou thème introduit par la première, puisque c'est le même contenu qui se trouve «élaboré ». On reste donc dans le «même », mais avec une phrase «autre »: la seconde phrase ou phrase élaborante est à la fois identique à et différente de la première ou phrase élaborée.

Il s'ensuit une tension entre le pôle de l'identité et celui de l' «altérité », qui peut entraîner un balancement soit du côté du «même », soit du côté de «l'autre », selon qu'on privilégie l'identité entre les deux phrases ou que l'on met l'accent plutôt sur le «détail» ou l'information qu'apporte la phrase élaborante. Dit encore autrement, le «détail», pris dans le sens d'information mineure, négligeable, conduit plutôt à souligner l'identité des deux phrases, alors que dans le sens de spécification nouvelle, il oriente vers la reconnaissance de la différence.

Cette tension est perceptible dans les définitions et caractérisations intuitives de la relation. Ainsi, chez Halliday (1994), dont nous redonnons ci-dessous avec un autre passage l'extrait déjà cité, émergent clairement les deux mouvements de sens opposé: ${ }^{6}$

\footnotetext{
${ }^{4}$ Le terme a été repris en français, mais en perdant au passage la transparence qu'il avait en anglais, ce que l'on peut regretter, étant donné les équivoques qu'il peut entraîner. Un terme tel que spécification semblerait nominativement meilleur, au moins pour les exemples canoniques.

${ }^{5} \mathrm{~S}=$ satellite (la deuxième phrase) et $\mathrm{N}=$ noyau (la première phrase).

${ }^{6}$ Voir à ce sujet le commentaire de Fabricius-Hansen et Behrens (2001: 3): 'This is a very broad and rather vague definition'.
} 
[...] one clause expands another by elaborating on it (or some portion of it) : restating in other words, specifying in greater detail, commenting or exemplifying (Halliday, 1994: 220);

In Elaboration, one clause elaborates on the meaning of another by further specifying or describing it. The secondary clause does not introduce a new element into the picture but rather provides a further characterization on one that is already there, restating it, clarifying it, refining it, or adding a descriptive attribute or comment. The thing that is elaborated may be the primary clause as a whole, or it may be just some part of it — one or more of its constituents (Halliday, 1994: 225).

D'un côté, on a l'idée que la phrase élaborante n'introduit pas véritablement quelque chose de nouveau (cf. les termes de restating in other words, restating it, clarifying it, refining it); de l'autre, on souligne qu'elle peut apporter vraiment du nouveau, en ce qu'elle caractérise en plus, ajoute un attribut descriptif ou un commentaire (cf. les termes de by further specifying or describing it, a further characterization, adding a descriptive attribute or comment). On retrouve également ces deux mouvements chez Hobbs (1979: 73) qui, après avoir souligné que 'at a sufficiently deep level the two sentences say the same thing', distingue deux cas:

-a- In the typical case, new information is conveyed by the second sentence, since there must be some reason for saying it again. This is why I have called the relation Elaboration rather than Paraphrase.

-b- However, I also mean to include under this headline such trivial moves as pure repetitions, repairs, tag questions, and the like. (Hobbs, 1979: 73)

Cette tension entre deux pôles opposés a pour résultat de rendre les définitions intuitives trop généreuses. Le pôle de l'identité conduit à intégrer - nous venons de le voir avec Halliday et Hobbs - les cas de paraphrases, de reformulations, de commentaires, d'exemplification, etc. Le pôle opposé, par l'intermédiaire de la notion trop vague de détail additionnel, de caractérisation nouvelle ouvre la porte à des brebis que l'on ne mettrait sans doute pas dans la bergerie d'Élaboration. C'est ainsi que, si l'on reprend la séquence (1) sous (6),

(6) Paul est tombé. Pierre l'a poussé.

on peut aussi dire que la deuxième phrase, en donnant la cause de la chute de Paul, apporte une information supplémentaire sur la première, qu'elle l'élabore en quelque sorte. ${ }^{7}$ On y verra pourtant plutôt la relation de Cause. Il en va de même pour (7):

\footnotetext{
${ }^{7}$ Les approches dites psychologiques ou cognitives ont bien montré d'ailleurs que les locuteurs auraient tendance à identifier ce type de relations en tant que relations causales avant tout. Ajoutons également que la plupart des travaux dans ce domaine n'incluent pas l'élaboration dans les relations de cohérence. Cf., entre autres, Sanders et al. (1992, 1993) et Gernsbacher et Givón (1995).
} 
(7) Paul s'est promené dans le parc. Le soleil brillait à travers le feuillage.

Elle représente un exemple de la relation Arrière-plan, mais on pourrait fort bien défendre l'idée que la deuxième phrase apporte un renseignement sur la première en ce qu'elle précise le temps qu'il faisait lors de la promenade de Paul dans le parc.

On retrouve également au niveau des caractérisations de la relation la trace de l'une ou l'autre orientation. L'orientation d'identité entre les deux phrases se reflète dans une des principales fonctions que reconnaît Hobbs à Elaboration, à savoir la fonction de clarification (cf. 'often for clarification', Hobbs, 1979: 73). Pour Hobbs, une des tâches premières d'Elaboration est d'éviter la mauvaise compréhension:

One function for Elaboration is obviously to overcome misunderstanding or lack of understanding. In procedural texts, when a sentence is insufficiently informative to determine the corresponding action, the reader or listener looks for an Elaboration next, and frequently finds it (Hobbs, 1979: 74).

Selon que l'on penche vers l'identité ou la différenciation, la relation d'Élaboration sera coordonnante ou subordonnante. L'identité conduit à envisager les deux phrases comme étant à un même niveau et présente donc Élaboration plutôt comme une relation coordonnante (c'est la position adoptée par Hobbs, 1990). Et, du coup, on fait de la relation Élaboration simplement un cas particulier de la relation Parallèle (cf. Hobbs, 1990: 95: 'the elaboration coherence relation is just the parallel relation when the similar entities ai and bi are in fact identical, for all i'; cf. aussi Kehler, 2004 qui place Elaboration parmi les relations de ressemblance mises en avant par Hume, les deux autres, comme on sait, étant Cause-effet et Contiguïté). Si l'accent est mis sur la différence des deux phrases, la relation sera subordonnante, l'une dominant l'autre (position adoptée par l'approche dite segmentée de la Segmented Discourse Representation Theory que nous verrons ci-après). Signalons qu'une telle asymétrie se trouve marquée chez Mann et Thompson (1988) par le statut de noyau (N) de l'élaborée et celui de satellite de l'élaborante (S).

En lien avec cette opposition coordonnant / subordonnant, on risquera ici la métaphore de la caméra pour faire apparaître d'une autre manière la différence de «vue " qu'entraînent les deux orientations. Dans le cas où élaborée et élaborante sont considérées comme étant de même niveau, c'est un simple changement de perspective: la première phrase est vue sous un autre angle, un autre point de vue. L'analyse faite par Hobbs (1979: 74) de l'exemple (8),

(8) Initialize. Set the stack pointer to zero, and set link variable P to ROOT. 
est à cet égard révélatrice, puisqu'elle fait clairement apparaître qu'une des fonctions possibles d'Elaboration est de présenter la «même pensée » d'un point de vue différent:

\begin{abstract}
Example -1 [ici 8] - comes from a published text [...], so the first sentence can't be a mistake that is corrected by the second. Why should the first sentence appear at all, if it can't lead to code? This suggests another function of Elaboration - it enriches the understanding of the listener by expressing the same thought from a different perspective. In algorithm descriptions, the first sentence typically describes the action in terms of the overall flow of control and the purposes of the algorithm. The second sentence describes it in terms of code. A single clause in English cannot easily support more than one point of view. (Hobbs, 1979: 74) ${ }^{8}$
\end{abstract}

Dans le cas où l'élaborée domine hiérarchiquement l'élaborante, il y a un Zoom soit sur l'ensemble de la phrase, soit seulement sur une de ses parties.

Au niveau des marqueurs et indices de la relation d'Élaboration, on ne retrouve pas véritablement une dissociation qui correspondrait aux deux pôles. Et cela pour une raison, principalement: il n'y a pas vraiment d'indices et de marqueurs qui soient décisifs pour l'identifier.' Ainsi, Scott et de Souza (1990) considèrent qu'il n'existe aucun item lexical pour la signaler: ${ }^{10}$ on ne peut la représenter qu'en insérant le satellite dans le noyau. Des propositions ont néanmoins été faites. C'est ainsi que des connecteurs sémantiquement orientés vers la précision, le détail, comme notamment, plus précisément, en particulier, etc., ont été cités pour être évalués sur le «terrain » dans le Projet ILF dirigé par Francis Cornish (Relations de cohérence et fonctionnement des anaphores). On ne saurait dire qu'ils reflètent le mouvement vers la différenciation. Par contre, les connecteurs avancés par Eggins (1994), à savoir in other words, that is (to say), to be more precise, in fact, I mean, manifestent clairement l'orientation vers l'identité. ${ }^{11}$

Il en va de même pour les coordonnées spatio-temporelles, puisque l'identité de lieu, de temps, d'agents, etc., que l'on trouve dans les présentations d'Élaboration, oriente fortement la relation vers le «même ». Fabricius-Hansen et Behrens (2001: 11) soulignent ainsi que:

8 Cette analyse nous semble erronée: ce n'est évidemment pas un changement de perspective qu'exprime la deuxième phrase, mais le moyen d'accomplir la première (cf. la possibilité d'avoir ici le gérondif pour la seconde en français: initialisez en plaçant ...). Dans Hobbs (1990: 97), l'analyse du même exemple nous semble beaucoup plus adéquate: 'The first sentence describes the role the operations play in the program as a whole. The second sentence gives the specifics of what has to be done'.

${ }^{9}$ Beaucoup de commentateurs soulignent ce fait (cf. Knott et Dale, 1994; Taboada et Mann, 2006).

${ }^{10}$ Ils donnent toutefois comme indice de reconnaissance de la relation d'Élaboration la présence d'une proposition relative.

${ }^{11}$ Voici les deux exemples qu'Eggins (1994: 105) propose en illustration:

a) Di's daughter was very sick. In fact, she needed a blood transfusion.

b) My daughter had jaundice. I mean she was as yellow as butter. 
E1 and e2 must belong to the same situation type (accomplishment / achievement, activity ... [...]) and have compatible argument structures. Their Agent referents must be identical, or more generally: referents having corresponding roles with respect to e 1 and e 2 must be identical, the temporal and spatial location of e 2 must be the same as or a subpart of the location of e1, etc. Somewhat sloppily, we can summarize these conditions as follows:

-a- situation type $(\mathrm{e} 1) \approx$ situation type (e2)

-b- agent $(\mathrm{e} 1) \approx \operatorname{agent}(\mathrm{e} 2)^{12}$

-c- time $(\mathrm{e} 1) \approx$ time $(\mathrm{e} 2)$

-d- $\operatorname{Loc}(\mathrm{e} 1) \approx \operatorname{Loc}(\mathrm{e} 2)$.

On signalera enfin, allant dans le même sens, que, dans la «grille d'analyse $»^{13}$ proposée pour saisir la relation d'Élaboration, Cornish place en septième point le facteur anaphorique, à savoir «la présence d'anaphoriques en $\mathrm{S}^{1}$ qui reprennent en parallèle soit le premier, soit le second argument du prédicat verbal de $\mathrm{S}^{\circ}$, soit les deux, soit encore le prédicat lui-même, voire même la proposition exprimée par $S^{\circ}$ dans sa totalité ».

Il faut encore mentionner - et ce sera le dernier point de cette présentation des deux tendances opposées qui articulent les approches intuitives - la question de la portée de l'Élaboration: l'Élaboration peut porter sur l'événement ou la proposition de la phrase élaborée - FabriciusHansen et Behrens (2001: 3), qui ont signalé ce point important, parlent alors de e[ventuality]-elaboration — ou seulement sur les actants de la proposition - il y a dans ce cas i[ndividual]-Elaboration. ${ }^{14}$ Ainsi, l'Élaboration présente dans la séquence (9),

(9) Il pleuvait. De grosses gouttes de pluie tombaient sur le sol (cf. Grabski, 2000)

est un cas d'e-Elaboration, alors que l'Élaboration réalisée par la seconde phrase de la séquence (10),

(10) Nous entrâmes dans un village. L'église était située sur une butte.

est un cas d'i-Elaboration, l'anaphore associative l'église (= l'église du village) marquant une Élaboration du seul participant 'un village' de la

12 C'est là que la problématique des relations de discours rencontre celle de l'anaphore. Voir Hobbs (1979 et 1990), Asher (1993: 267) et les travaux récents de Cornish sur la question.

${ }^{13}$ Nous trouvons également dans cette grille, entre autres, les temps verbaux, certains connecteurs, les relations lexicales telles que la méronymie et l'hyponymie (projet ILF). Voir le tableau 1 dans Cornish (ici-même).

14 Voir aussi à cet égard l'article de Prévot et al. (ici-même). 
première. ${ }^{15}$ On voit que, dans l'optique d'une Élaboration d'identité, une telle différence n'est pas à faire : il ne peut s'agir que d'e-Elaboration, l'autre Élaboration ne permettant pas par avance de conclure à l'identité des deux phrases. Dans la suite de ce travail, nous ne nous occuperons que d'eElaboration, les différentes conceptions qui seront analysées ne valant que pour ce type d'Élaboration.

\section{LA RST: EN TRANSITION}

Ceux qui sont tournés vers le pôle «identité » de la relation n'ont guère besoin de préciser plus avant la relation d'Élaboration. Il n'en va évidemment pas de même pour ceux qui sont tournés vers le pôle « différenciation »: là il s'agit bien de dire quel est l'élément nouveau, quel est le détail additionnel apporté par la phrase élaborante. On ne peut en effet en rester au «détail », à la «précision supplémentaire » apportée sur la phrase élaborée, sinon, comme nous l'avons déjà souligné ci-dessus avec les exemples Paul est tombé. Pierre l'a poussé et Paul s'est promené dans le parc. Le soleil brillait à travers le feuillage, on court le risque de devoir accueillir comme Élaboration des relations qui intuitivement sont perçues comme étant des relations différentes.

Mann et Thompson (1987 et 1988), dans le cadre de la RST, proposent une définition (c'est la partie 'contraintes sur $\mathrm{N}+\mathrm{S}$ ' de la définition) qui remédie au défaut que nous venons de signaler, parce qu'elle indique quels sont précisément les types d'information nouvelle ou détails additionnels qui peuvent être apportés par la phrase élaborante. Elle le fait en énumérant six types d'opposition dont la phrase élaborée est le premier membre et la phrase élaborante le second:

$\mathrm{S}$ presents additional detail about the situation or some element of subject matter which is presented in $\mathrm{N}$ or is inferentially accessible in $\mathrm{N}$ in one our more of the ways listed below. In the list, if $\mathrm{N}$ presents the first member of any pair, then $\mathrm{S}$ includes the second:

set: member

abstraction: instance

whole: part

process: step

\footnotetext{
${ }^{15}$ Les relatives appositives représentent un autre cas «standard » d'i -Elaboration. Notons toutefois que Fabricius-Hansen et Behrens (2001: 6) illustrent la relation d'Élaboration canonique à l'aide d'un exemple qui, nous semble-t-il, ne relève pas de ce cas de figure: «That Leni herself is a 'Particlist' is something she proves by gathering up all the bread crumbs from her plate and putting them into her mouth ». Le problème avec cet exemple est que le verbe prove ainsi que le gérondif by gathering up conduisent plutôt à une relation du type Indice-preuve. De plus, l'Élaboration, si Élaboration il y a, ne se fait pas entre événements, mais entre conditions. Il est d'ailleurs significatif que les auteurs optent pour une nouvelle étiquette afin de décrire cet exemple: ils parlent de Causal-instrumental elaboration. Nous reprendrons sous un autre angle cet exemple ci-dessous.
} 
object: attribute

generalization: specific (Mann et Thompson, 1988: 273)

L'avantage de cette définition est certain, puisque des noms plus clairs sont mis sur les manières d'établir la relation d'Élaboration. Il y a une identification positive des « différences » de la phrase élaborante par rapport à la phrase élaborée, en même temps que se trouve reconnue ce qui les relie. On voit qu'une telle définition n'est pas dirigée vers l'identité et donc des cas «paraphrastiques » comme la reformulation ou les répétitions, mais qu'elle se situe clairement du côté qui souligne la différenciation entre les deux dans la mesure où précisément la phrase élaborante, dans les six soustypes d'Élaboration présentés, est à chaque fois différente de la phrase élaborée et a aussi un statut différent. Trois défauts ou difficultés subsistent néanmoins. En premier lieu, pourquoi garder Élaboration, alors que l'on dispose de six relations nommées et différenciables ? Élaboration pourrait alors représenter un grand groupe générique ou superordonné de relations, comme, par exemple, Expansion. Les auteurs répondent de deux manières à cette objection. Une première réponse se trouve déjà dans la définition: comme on peut avoir 'one or more of the ways listed below', c'est-à-dire comme on peut avoir plusieurs sous-types ensemble, on ne saurait se priver d'Élaboration. La deuxième réponse prévoit directement l'objection faite: 'the subtypes are not treated as separated relations because in many cases in text they are not distinguishable' (Mann et Taboada, 2005: 9) (cf. aussi Knott et al., 2001).

Il faut donc conserver Élaboration. Si elle surmonte la première difficulté, une telle réponse est aussi l'indice d'une autre difficulté.

Le deuxième défaut de la définition de Mann et Thompson est que les six sous-types proposés manquent d'efficacité: on n'arrive pas toujours à savoir de quel(s) sous-type de relation il s'agit. Pourquoi les distinguer alors s'ils ne se révèlent pas efficaces, étant donné que (cf. notre point de départ) c'est au lecteur de faire le bridging, c'est-à-dire de reconnaître le lien de cohérence à établir entre les deux phrases? Et, du coup, on peut réclamer au titre de cette inefficacité une autre définition qu'une conjonction de relations inopérantes.

Troisième difficulté, enfin, chaque relation pose elle-même le problème de sa propre définition. C'est de là, nous semble-t-il, que vient l'obstacle de les reconnaître in situ. Quelle est exactement la différence entre set: member, generalization: specific et abstraction: instance? Comment faut-il comprendre le lien entre objet et attribut? A ce propos, l'exemple (11) donné par Mann et Taboada (2005: 9) comme illustration de ce sous-type n'est pas tellement paradigmatique d'une telle relation dans la mesure où il pourrait tout aussi bien s'agir du sous-type partie-tout que du sous-type objet-attribut. La première phrase peut en effet apparaître comme introduisant un objet ou un tout et les différentes phrases qui l'élaborent ensuite comme des attributs ou des parties: 
(11) 'City', in Sweden, will be the site of the 1969 International Conference on Computational Linguistics, September 1-4. It is expected that some 250 linguists will attend from Asia, West Europe, East Europe including Russia, and the United States. The conference will be concerned with the application of mathematical and computer techniques to the study of natural languages, the development of computer programs as tools.

Il ne subsiste finalement de Mann et Thompson que le côté type (qui domine les six sous-types) et qui reste totalement intuitif, à savoir celui que ' $\mathrm{S}$ presents additional detail about the situation or some element of subject matter which is presented in $\mathrm{N}$ or inferentially accessible in $\mathrm{N}^{\prime}$. Ce qui représente quand même un élément important dans la mesure où on s'aperçoit que ce qui est commun aux six sous-types, c'est que la première phrase, l'élaborée, est, dans les six cas, d'une manière ou d'une autre, plus grande que l'élaborante, conçue comme l'englobée, l'ordre allant du plus grand au plus petit et jamais dans le sens inverse.

\section{DEFINITIONS FORMELLES DE LA RELATION D'ELABORATION}

Le passage aux définitions formelles représente une étape de plus pour essayer, soit dans le sens de l'identité, soit dans celui de la différenciation, de cerner avec des instruments sémantiques plus rigoureux l'intuitive relation d'Élaboration. On examinera, comme annoncé, d'abord la conception de Hobbs, puis celle de la SDRT. La première, on le verra, est penchée vers l'identité, la seconde entend plutôt saisir la différence entre phrase élaborée et phrase élaborante.

\subsection{Définitions de Hobbs (1979 et 1990)}

Hobbs (1979 et 1990) recourt à l'inférence et stipule, comme le montrent les définitions de 1979 et de 1990 données ci-dessous que la relation d'Elaboration se définit par le fait que les deux phrases, aussi bien l'élaborée S0 que l'élaborante S1, donnent lieu à une inférence commune:

Elaboration: $\mathrm{S} 1$ is an Elaboration of $\mathrm{S} 0$ if a proposition $\mathrm{P}$ follows-from the assertions of both $\mathrm{S} 0$ and $\mathrm{S} 1$ (but $\mathrm{S} 1$ contains a property of one of the elements of $\mathrm{P}$ that is not in S0). (Hobbs, 1979: 73).

Elaboration: Infer the same proposition P from the assertions of $\mathrm{S}^{\circ}$ and $\mathrm{S} 1$ (Hobbs, 1990: 95).

Dans la définition de 1990, ne figure plus le contenu de la parenthèse de 1979. L'exigence de nouveauté n'est donc plus définitoire, mais le fait de l'avoir mise entre parenthèses en 1979, était déjà l'indication qu'il ne s'agissait pas d'un trait nécessaire. La position de 1990 n'est donc pas 
différente de celle de 1979. En 1990 comme en 1979, Hobbs admet que le plus souvent ou dans les cas typiques il y a du nouveau. S'il ne l'intègre pas dans la définition de 1990, ou la met entre parenthèses dans celle de 1979, c'est pour pouvoir intégrer les répétitions et autres reformulations: 'Frequently, the second segment adds crucial information, but this is not specified in the definition since it is desirable to include pure repetitions under the headline of elaboration' (Hobbs, 1990: 96).

La première chose qu'il faut noter est que la définition logique donnée n'est pas immédiatement transparente par rapport à l'intuition que l'on peut avoir de la relation Élaboration. On comprend cependant ce qui la motive: elle est destinée à représenter qu'une phrase élaborante ne sort pas de la situation dénotée par la phrase élaborée, qu'il s'agit en fait de la même situation ou proposition $\mathrm{P}$, avec une ou des informations apportées (les « détails ») par $\mathrm{S}^{1}$ sur des éléments de $\mathrm{P}$ et qui ne sont pas données par $\mathrm{S}^{\circ}$.

On voit aussi qu'elle est tournée vers le côté identité ou " mêmitude » des deux phrases, puisque le critère définitionnel décisif est ce qui unit les deux phrases et non ce qui les sépare: si Hobbs recourt à l'inférence commune $\mathrm{P}$ pour définir l'élaboration, c'est qu'il entend saisir par là le fait que les deux phrases à un certain niveau (celui de $\mathrm{P}$ ) sont équivalentes, c'est-à-dire, comme nous l'avons signalé déjà ci-dessus, que 'at a sufficiently deep level, the two sentences say the same thing' (Hobbs, 1979: 73).

Sa non transparence initiale exige que l'on montre comment elle fonctionne sur un exemple précis. Hobbs (1979 et 1990) se sert d'une instruction d'itinéraire pour illustrer ce fonctionnement:

(12) Go down Washington Street. Just follow Washington Street three blocks to Adams Street.

Voici son analyse:

It is important that anyone trying to follow these directions recognize the second sentence as an Elaboration and not as the next instruction. The pattern is recognized by inferring a 'going' from 'follow' and matching the paths — Washington Street from the two sentences. Then 'to Adams Street' elaborates on the unstated end point of the 'going' in the first sentence, and 'three blocks' adds measure to its paths (Hobbs, 1979: 74).

La proposition commune $\mathrm{P}$ que l'on infère de l'assertion de $\mathrm{S} 0$ et de $\mathrm{S} 1$ est l'instruction de se rendre à un certain endroit de Washington Street, instruction qui est commune à $\mathrm{S} 0$ et $\mathrm{S} 1$ (Hobbs souligne bien dans son commentaire qu'il ne faut pas interpréter la deuxième phrase comme une nouvelle instruction). Il faut, pour construire cette inférence commune, d'abord accepter que follow de S1 implique going (que 1'on a dans S0), ensuite établir par l'intermédiaire de Washington Street présent dans les 
deux phrases que la «localisation» du «going » est la même et que l'endroit où il faut aller qui reste non spécifié dans $\mathrm{S} 0$ se trouve déterminé par l'indication Adams Street de S1 et, enfin, reconnaître que la longueur du trajet à effectuer, non précisée dans S0, l'est aussi dans S1 par l'indication three blocks («trois pâtés d'immeubles »).

Il faut encore noter que S1, en précisant qu'il ne faut descendre la Washington Street que sur trois rues (pâtés d'immeubles), jusqu'à l'Adams Street, exclut bien entendu l'interprétation globalisante de S0, à savoir qu'il faut descendre toute la Washington Street, que le lecteur serait en droit d'établir s'il n'y avait pas S1 (voir la fonction déjà évoquée ci-dessus que prête Hobbs à l'Élaboration, à savoir celle d'éviter le malentendu ou le manque d'information).

Deuxième point à noter: le rapport avec les répétitions et les reformulations. Cette définition en termes d'inférence commune a l'avantage, signalé par Hobbs lui-même, de pouvoir englober à côté des cas typiques comme l'exemple d'instruction d'itinéraire, où S1 apporte des informations sur des éléments non spécifiés ou «non élaborés » de S0, les cas de répétition ou de reformulation, où $\mathrm{S} 1$ n'apporte pas d'informations qui soient nouvelles par rapport à $\mathrm{S} 0$. La raison d'être des reformulations correspond bien souvent à la fonctionnalité que prête Hobbs à la relation d'Élaboration: soit celle de prévenir la mauvaise compréhension, de clarifier le propos pour l'auditeur, etc., soit encore, comme déjà signalé, celle de présenter le même contenu selon des perspectives ou points de vue différents. Le point essentiel, à notre avis, est que l'inclusion des situations de répétition et de reformulation signifie l'inclusion de phrases en relation d'équivalence logique, où S0 implique S1 et S1 implique S0.

En troisième lieu, les exemples du type de l'instruction d'itinéraire pourraient être caractérisés logiquement de façon plus précise que celle de donner lieu à une inférence commune. $\mathrm{Si}$ on accepte l'analyse faite par Hobbs de l'exemple (12) donné ci-dessus:

(12) Go down Washington Street. Just follow Washington Street three blocks to Adams Street.

on peut établir une relation d'implication unilatérale entre S0 et S1: S1 implique S0, mais non réciproquement: descendre la Washington Street sur trois pâtés d'immeubles jusqu'à Adams Street implique descendre la Washington Street jusqu'à un certain point, mais l'inverse bien entendu ne tient pas. On comprend pourquoi Hobbs n'a pas retenu une telle formulation, si on la rattache à notre point précédent: cela revient à exclure les cas d'implication réciproque ou d'équivalence, donc les répétitions et autres reformulations de contenu identique, que Hobbs tient tout particulièrement à intégrer dans les cas d'Elaboration. 
Cela a une deuxième conséquence, celle d'entraîner la subordination de $\mathrm{S} 1$ par rapport à S0. Or, et c'est là le quatrième point de notre présentation, la définition hobbsienne de la relation d'Elaboration en présentant S0 et S1 comme donnant lieu à une inférence commune $\mathrm{P}$ les présente à un même niveau et les donne donc plutôt, conséquence déjà signalée ci-dessus, comme coordonnées que comme $\mathrm{S} 1$ subordonnée à S0.

Signalons encore que la définition «inférentielle » de Hobbs connaît des difficultés à rendre compte des exemples du type de Kamp-Rohrer (1983) comme (13):

(13) L'an dernier, Jean escalada le Mont Cervin. Le premier jour, il monta jusqu'à la cabane H. Il y passa la nuit. Ensuite il attaqua la face Nord. Douze heures plus tard il arriva au sommet. (Kamp et Rohrer, 1983)

Chacune des phrases qui suit la première apporte un «détail » sur l'escalade de Jean, puisque chacune d'entre elles présente une étape de cette escalade. Mais, prises séparément avec la première, elles ne satisfont pas à la définition de Hobbs, puisque, isolément, elles ne permettent pas en relation avec la première d'inférer une proposition commune $\mathrm{P}$. Tout simplement parce qu'elles ne précisent ou ne complètent qu'une partie du processus dénoté par la première. De L'an dernier, Jean escalada le Mont Cervin et de Le premier jour, il monta jusqu'à la cabane $H$, on ne peut inférer une proposition identique, parce que la seconde, n'étant qu'une « portion » de la première, ne peut préciser ou saturer un ou des éléments de la phrase qui renvoie au processus global. Ce n'est que si l'on met l'ensemble des phrases élaborantes en relation avec la première que l'on peut appliquer la définition de Hobbs, puisque l'on peut alors inférer, via l'identité du processus global obtenu, une proposition commune telle que Jean a escaladé le Mont Cervin pour laquelle les phrases qui suivent la première spécifient les différentes étapes de la montée laissées sous-déterminées par la première.

Nous avons vu que le fait de privilégier l'aspect d'identité de la relation d'Élaboration laisse dans l'ombre l'aspect différentiel entre la phrase élaborée et la phrase élaborante. Autrement dit, la question du type d'information additionnelle éligible pour la relation d'Élaboration n'est pas réglée ou ne reste qu'un ... détail. La définition de la SDRT, aussi bien dans la version d'Asher (1993) que celle d'Asher et Lascarides (2003), apporte, comme nous allons le voir, une réponse à cette question.

\subsection{SDRT: N. Asher (1993)}

Nous commencerons par la définition d'Asher (1993: 267):

DEFINITION: Elaboration ( $a \downarrow b$ ) iff ( $a b v$ (for every e ME(b) there is an $\mathrm{e}^{\prime} \mathrm{ME}(\mathrm{a})$ such that e is a part of $\left.e^{\prime}\right) \& b$ is more complex than $a$. 


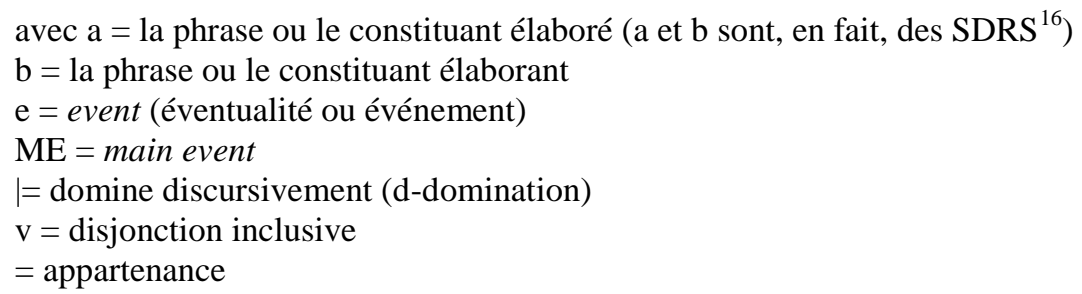

La technicité de cette formule définitoire demanderait des explicitations ${ }^{17}$ qui nécessiteraient une incursion dans le domaine de la SDRT qui nous entraînerait beaucoup trop loin (cf. Busquets et al., 2001 et Bras et Le Draoulec, ici-même). Il nous suffira pour notre propos de faire ressortir les points sémantiques essentiels de cette définition et nous laisserons donc volontairement de côté toutes les définitions formelles (données page 300) des différents éléments qui la constituent, l'essentiel en l'occurrence étant de comprendre quels sont les traits sémantiques qu'assigne la SDRT à la relation d'Élaboration. L'affaire est d'autant plus nécessaire que bien souvent les définitions ne sont pas mises à l'épreuve sur des exemples. Trois conditions sont postulées:

-i- a d-domine (domine discursivement) b

-ii- l'événement dénoté par b est une partie de l'événement dénoté par a.

-iii- une condition de complexité: $b$ doit être plus complexe que a.

La condition -iii-, étant donné la coordination, est toujours présente, alors qu'il n'en va pas ainsi des deux premières conditions, puisqu'elles forment une disjonction inclusive.

Nous nous arrêterons surtout sur les deux premières conditions, puisque la troisième, la condition de complexité, vise essentiellement, nous semble$\mathrm{t}-\mathrm{il},{ }^{18}$ à rendre compte de ce que la phrase élaborante $\mathrm{b}$ doit être plus riche $\mathrm{du}$ point de vue informationnel que la première, autrement dit qu'elle précise ou apporte des informations non contenues dans la phrase élaborée et qu'au final elle est donc plus précise, plus spécifiée ou plus complexe que l'élaborée. On n'est donc pas loin de la "parenthèse » de la définition de Hobbs (1979) sur l'apport par S2 d'une propriété non contenue dans S1 (cf. Asher et Lascarides, 2003: 162: 'in addition, Asher (1993) argues that if

\footnotetext{
16 Une SDRS est, en gros, un constituant complexe (contenu propositionnel symbolisé $\pi$ où $\pi$ est une étiquette et $\mathrm{K}$ le constituant qu'elle désigne). Ainsi, une relation est subordonnante ssi elle peut introduire une SDRS complexe. Voir à ce propos Busquets et al. (2001).

${ }^{17}$ Comme l'ont relevé Fabricius-Hansen et Behrens (2001: 4), il manque une parenthèse fermante, ce qui n'est pas pour faciliter la compréhension.

${ }^{18}$ Voir la définition formelle donnée par Asher (1993: 300) que traduisent ainsi FabriciusHansen et Behrens (2001: 4): ' $b$ is more complex than a iff either the set of main events established in $b$ is bigger than the set of main events established in $a$ or the set of constituents of $\mathrm{b}$ is bigger than the set of constituents of a [where $\mathrm{a}$ and $\mathrm{b}$ are SDRSes]'.
} 
Elaboration (a,b) holds, then $K b$ describes in more detail some part of $K b^{\prime}$ ). Par domination discursive (d-domination), comme il ressort de la définition donnée ci-dessous, il faut entendre que la phrase élaborée est hiérarchiquement supérieure à la phrase élaborante, qu'elle la subsume à la manière d'un hyperonyme qui domine lexicalement ses hyponymes ${ }^{19}$ et qu'entre la phrase élaborée et la phrase élaborante s'établit une hiérarchie sortale (type / sous-types) qui trouve son origine dans un savoir encyclopédique partagé:

The definition of $d$-domination assumes a sort hierarchy in which there is a subsort relation that is a partial ordering on the set of sorts. The sort hierarchy will cover both events types and object types. The sort hierarchy is part of a fixed knowledge base, in which is to be encoded common sense word knowledge (Asher, 1993: 300).

L'exemple suivant tiré du corpus Cornish (projet ILF) permet d'illustrer cette d-domination:

(14) Au petit matin, hier, vers 5h30, un homme de 28 ans, passablement éméché, a importuné des policiers qui procédaient à un contrôle routier sur les allées Jean-Jaurès à Toulouse. Celui-ci a menacé les fonctionnaires avec sa cannette de bière, tout en émaillant ses propos de quelques noms d'oiseaux. (La Dépêche du Midi, 9/07/03).

Les deux événements correspondant aux deux propositions élaborantes de la seconde phrase, à savoir «menacer les fonctionnaires avec sa cannette de bière » et «émailler ses propos de quelques noms d'oiseaux », apparaissent bien comme étant des sous-types de l'événement, importuner des policiers, de la première phrase, puisque menacer quelqu'un avec une canette de bière et lui dire des noms d'oiseau représentent bien des manières d'importuner quelqu'un. Il y a donc bien subsomption entre l'événement ' $\mathrm{X}$ importuner $Y^{\prime}$ ' et ' $X$ menacer ... $Y$ ' / ' $X$ dire des noms d'oiseau à $Y$ ', en supposant bien entendu, ce qui est le cas dans l'exemple, que $\mathrm{X}$ et $\mathrm{Y}$ sont les mêmes. ${ }^{20}$

Le fait de postuler une condition de d-domination ou subsomption rend compte de ce que le rapport entre $a$ et $b$ est un rapport d'implication unilatérale: $b$ implique $a$, mais a n'implique pas $b$ : ' $a$ d-dominates $b$ iff $a$ subsumes $\mathrm{b}$ [relative to a background knowledge base with a sort structure] or (b entails a, and not a entails b)' (Asher, 1993: 300).

Alors que Hobbs, pour un exemple tel que celui que nous venons de citer, en reste à l'inférence commune que l'on peut tirer des assertions de a et de b («un homme éméché a importuné des policiers ») et ne va pas,

${ }^{19}$ En note, Asher (1993: 310) souligne explicitement cette parenté: 'A similar notion of domination finds its way into knowledge bases for is a-hierarchies'.

${ }^{20}$ Rappelons que c'est pour cette raison que les problèmes anaphoriques ont partie liée avec la question des relations de cohérence et que, comme l'a montré Cornish (à par.), il y a complémentarité. 
comme nous l'avons souligné (cf. supra), jusqu'à caractériser la relation en termes d'implication unilatérale, Asher aboutit à un tel résultat parce que, contrairement à Hobbs, tourné plutôt vers l'identité, il met au premier plan la différence de statut hiérarchique des deux événements et peut donc rendre compte du rapport implicationnel unilatéral entre a et b:

$\mathrm{X}$ menace $\mathrm{Y}$ avec une canette de bière $\rightarrow \mathrm{X}$ importune $\mathrm{Y}$

$\mathrm{X}$ importune $\mathrm{Y} \longrightarrow \mathrm{C} \longrightarrow \mathrm{X}$ menace $\mathrm{Y}$ avec une canette de bière

Ajoutons que cette d-domination conduit à appréhender la relation d'Élaboration comme une relation subordonnante. La relation d'Élaboration, note Asher (1993: 266) 'does not introduce just a two-place relation, but rather a complex condition in which one constituent discourse dominates or $d$-dominates another - much in the same way that a more general topic dominates a more specialized topic in a taxonomy of some domain'.

Ce n'est pas pour autant que la facette «identité » de la relation d'Élaboration se trouve oubliée dans sa définition de la relation de $d$ domination. En effet, Asher insiste sur le fait que la phrase qui «d-domine » est en quelque sorte le topique de l'autre: ' $a$ d-dominates b' means that a is a semantic label for $b$; it holds just in case a acts as a summary or topic statement of b' (Asher, 1993: 267).

La deuxième condition, d'orientation 'more narrative', stipule que 'one event is part of or a subevent of another event' (Asher, 1993: 267). On peut reprendre ici l'exemple de l'escalade du Mont Cervin (exemple 13 repris ici sous 15 ), où l'on voit bien que les quatre phrases qui suivent la première sont des élaborations de celle-ci, puisqu'elles dénotent chacune un événement qui est une partie ou un sous-événement de l'événement rapporté par la première:

(15) L'an dernier, Jean escalada le Mont Cervin. Le premier jour, il monta jusqu'à la cabane H. Il y passa la nuit. Ensuite il attaqua la face Nord. Douze heures plus tard il arriva au sommet. (Kamp et Rohrer, 1983)

Précisons encore que le trait de subordination et le trait topical avancés pour la d-domination se laissent appliquer sans difficulté à cette condition de 'partie-de', dans la mesure où un tout peut être dit dominer ses parties (la partie n'est pas au même niveau hiérarchique que le tout) et que si l'on passe du tout aux parties, on ne sort pas du tout, mais on ne fait que développer une partie du tout topical introduit.

La question que posent les deux premières conditions de subsomption et de 'partie-de' est celle de leur rapport: 'It is not very clear to us', concluent Fabricius-Hansen et Behrens (2001: 5), 'how the two disjunct clauses of the definition hang together'. C'est ce problème que nous allons aborder avant 
d'examiner les conséquences de la définition d'Asher sur le recrutement des exemples d'Élaboration.

La définition d'Asher unit bien, comme nous l'avons noté supra, les deux conditions en question par le connecteur logique de disjonction inclusive, ce qui a pour conséquence de prévoir les trois cas possibles -a-, -b- et -c-:

-a- le premier membre est seulement vrai: c'est le cas où il n'y a que $d$-domination ou subsomption et non relation de type 'partie-de' ou de 'sous-événement'.

-b- le second seulement est vrai: on n'a alors que la relation de type méréologique et non celle de subsomption.

-c- les deux membres sont vrais en même temps, c'est-à-dire que l'exemple doit vérifier à la fois la relation de subsomption ou de d-domination et celle de partietout.

Rencontre-t-on ces trois possibilités? Pour -a-, point de difficultés, nous l'avons vu avec l'exemple de l'homme qui importune les policiers en les menaçant d'une canette de bière. Pour -b- non plus: l'exemple de l'escalade du Mont Cervin montre bien que l'on peut avoir un rapport de type 'partietout' sans qu'il y ait subsomption entre les deux constituants, puisque, comme déjà signalé ci-dessus, « $\mathrm{X}$ monter à la cabane $\mathrm{H}$ » ou « $\mathrm{X}$ passer la nuit à la cabane $\mathrm{H}$ » n'implique pas « $\mathrm{X}$ escalader le Mont Cervin ».

Pour -c-, les choses sont plus délicates: est-il possible d'avoir à la fois $d$ domination et relation partie-tout ou relation de sous-événement? Un exemple donné par Asher et Lascarides (2003: 462) comme illustration de la définition d'Élaboration semble bien correspondre à la condition -a-,

(16) Max had a lovely meal last night. He ate lots of salmon.

puisque, comme le donne à penser l'analyse des auteurs - on y reviendra He ate lots of salmon, d'un côté, implique bien ou est subsumée par Max had a lovely meal last night et, de l'autre, apparait, en tant que plat, comme n'étant qu'une partie du repas, donc qu'une partie temporelle ou sousévénement du repas en question. Si on y regarde de plus près, on voit qu'il y a une faille dans l'analyse. Voici comment. Admettons l'hypothèse que le repas ait été effectivement constitué de plusieurs plats, dont le plat de saumon. A ce moment-là, pour que l'on puisse dire, dans l'optique de la $d$ domination ou subsomption, que le repas était excellent, il ne faut pas que tous les autres plats ou la majorité des autres plats, par exemple, aient été mauvais. ${ }^{21} \mathrm{Ou}$, si l'on tient quand même, toujours dans une veine subsomptive, à dire que le plat de saumon a fait que le repas a été fameux, il faut faire abstraction des autres plats, c'est-à-dire il faut nier la dimension partie-tout ou 'sous-événement' pour ne retenir que la seule relation «manger du saumon (en quantité) », c'est « bien manger » ou « faire un bon

${ }^{21}$ Ce qui par les temps qui courent n'est absolument pas exclu. Parole de gastronomes ! 
repas ». On le voit, les deux dimensions ne peuvent être maintenues en même temps. Si on privilégie la relation partie-tout, la subsomption doit céder; si, au contraire, on opte pour la relation d-domination, c'est la relation 'partie-de' qui disparaît. Il paraît donc clair que l'on ne peut avoir la possibilité -c-, c'est-à-dire la conjonction de la $d$-domination ou de la relation de 'sous-types' et de la relation 'partie-de' ou de 'sousévénement'. 22

C'est dire du même coup qu'en fait il n'y a pas disjonction inclusive, mais disjonction exclusive: c'est soit la subsomption, soit la relation 'partiede' ou de sous-événement qui l'emporte. On notera que, dans le premier cas, la phrase élaborante dénote le même événement que celui de la principale nous rejoignons ici Hobbs - alors que, dans le second, elle ne renvoie qu'à une partie de celui-ci et donc est plutôt orientée vers la différenciation. ${ }^{23}$

On voit par là-même que la conception d'Asher permet de rendre compte des emplois que maîtrise la définition de Hobbs, mais en excluant par la condition de complexité les cas de paraphrases, de répétitions et de simples reformulations. Et elle a l'avantage, par le deuxième membre de la disjonction exclusive, de rendre également compte des cas de type 'partiede' ou de 'sous-événement' que ne peut prendre en charge, comme nous l'avons vu supra, la théorie de l'inférence commune de Hobbs.

Il reste néanmoins deux difficultés qui altèrent un peu la disjonctive image formelle ainsi tracée. Premièrement, le même reproche fait ci-dessus à Mann et Thompson peut être adressé à la définition disjonctive: pourquoi conserver une seule relation et ne pas en distinguer tout de suite deux, qui correspondent à la relation de subsomption et à celle de 'partie-de' ou de 'sous-événement'? Deuxièmement, et plus grave, avec le second membre de la disjonction, on court le risque de devoir accueillir des emplois qu'une approche intuitive ne classerait sans doute pas dans la catégorie des élaborations.

Il en va ainsi de l'exemple qui sert de base à l'analyse de FabriciusHansen et Behrens (2001) consacrée à la relation d'Élaboration entrevue dans une perspective interlinguistique allemand-anglais-norvégien et dans un cadre intraphrastique ${ }^{24}$ présentant deux propositions reliées par un connecteur. Le connecteur de départ est l'allemand indem et l'exemple allemand avec ses traductions anglaise et française est le suivant:

\footnotetext{
${ }^{22} \mathrm{Si}$ l'on interprète de façon « lâche » la relation partie-tout pour y inclure, par exemple, la relation 'objet-propriété', alors la situation -c- est possible. Mais dans ce cas les exemples qui y correspondent sont aussi ceux de la situation -a- comme l'exemple de l'hommeéméché-qui-menace-les-policiers.

${ }^{23}$ Fabricius-Hansen et Behrens (2001) présentent la définition de Behrens (1998) qui ne s'applique qu'à la première partie (celle où l'événement reste le même) de la définition d'Asher.

${ }^{24}$ Cela suppose, bien entendu, que l'on accepte que les relations de discours conviennent aussi en dehors des relations entre phrases indépendantes.
} 
(17) Das Leni selbst eine «Partikularistin » ist, beweist sie täglich, indem sie sämtliche Brötchenkrümel vom Teller aufliest und in den Mund steckt (Fabricius-Hansen et Behrens, 2001: 6)

(17') That Leni herself is a «Particlist» is something she proves daily by gathering up all the bread crumbs from her plate and putting them into her mouth.

$17^{\prime \prime)}$ Que Leni elle-même est "spéciale" se prouve quotidiennement par le fait qu'elle ramasse toutes les miettes de pain dans l'assiette et les met en bouche.

L'analyse de Fabricius-Hansen et Behrens conclut à la relation d'Élaboration entre la proposition principale et la proposition introduite par indem, parce qu'elle s'appuie sur le fait que la proposition indem sie sämtliche Brötchenkrümel vom Teller aufliest und in den Mund steckt ne fait que spécifier un sous-événement (subevent) de la proposition principale en indiquant par quel moyen se prouve le fait que Leni est quelqu'un de vraiment particulier. Fabricius-Hansen et Behrens analysent en effet (dans le carde formel de la DRT) le premier événement comme étant un événement causatif complexe (causative event complex) qui contient implicitement et de façon non spécifiée le sous-événement qui est à l'origine du résultat, c'est-à-dire de ce que la preuve est faite du caractère de Leni. La phrase introduite par indem ou celle introduite par by gathering vient donc élaborer ou spécifier ce sous-événement laissé indéterminé dans la phrase matrice. 'The subclause identifies', concluent Fabricius-Hansen et Behrens (2001: 30), 'the implicit causal-instrumental subevent of a structured causative event complex established in the matrix clause'.

Ce type de situation n'est pas restreint au cadre intraphrastique et aux connecteurs. La même relation peut apparaître avec des phrases indépendantes, non reliées par un connecteur. Dans la séquence (18):

(18) Paul a éteint le feu. Il a pissé dessus.

la seconde phrase explicite en effet par quel moyen Paul a éteint le feu et on est donc en droit de parler d'Élaboration. Ce qu'il faut noter aussi bien pour l'exemple en indem que pour l'exemple d'éteindre le feu, c'est qu'il n'y a pas de subsomption possible: "x éteindre le feu », par exemple ne d-domine d'aucune manière « $\mathrm{x}$ pisser sur le feu » («x pisser sur le feu » n'implique évidemment pas «x éteindre le feu ») et il n'y a donc pas identité d'événement entre les deux. Mais ce qu'il faut bien voir et qui fait que l'on puisse être gêné pour parler d'Élaboration pour ce genre de relation, c'est que «pisser sur le feu » n'est pas une partie de «éteindre le feu » comme, par exemple, «monter le premier jour jusqu'à la cabine $\mathrm{H}$ », est une partie de l'événement «escalader le Mont Cervin ». La preuve, c'est qu'il n'est pas inclus temporellement dans l'événement d'éteindre le feu, puisque sa 
durée semble plutôt coïncider avec celle d'éteindre le feu. Il s'agit donc de deux événements différents de ce point de vue-là et si Fabricius-Hansen et Behrens parlaient de sous-événement pour le second, ce n'est pas dans le sens d'événement qui forme une partie (temporelle) du premier, mais dans le sens d'événement qui est impliqué comme événement différent dans une structure comprenant d'autres événements. A ce moment-là, on l'aura compris, la porte est ouverte également pour y ranger des relations comme celle de Cause, pourtant jamais placée sous le chapeau d'Élaboration. En effet, à propos de l'exemple canonique:

(19) Paul est tombé. Pierre l'a poussé.

on peut aussi dire que Pierre l'a poussé spécifie le sous-événement de la structure complexe que l'on peut assigner à la première phrase, sousévénement resté implicite et non déterminé, à savoir ce qui a causé la chute de Pierre.

Si l'on entend maintenir le deuxième membre de la définition disjonctive d'Asher (1993), il convient, pour éviter la difficulté que nous venons d'exposer, de préciser ce qu'on entend par 'partie-de' ou 'sousévénement'. Cette clarification se trouve apportée dans Asher et Lascarides (2003), qui constituera la dernière étape de notre parcours « élaborant».

\subsection{SDRT: Asher et Lascarides (2003)}

Cette dernière étape sera plus rapide que la précédente, puisque bon nombre d'éléments d'Asher (1993) se trouvent repris dans Asher et Lascarides (2003). C'est ainsi qu'ils reprennent la notion de subordination ${ }^{25}$ et qu'ils conservent la notion de subsomption (ils ne parlent plus de d-domination, mais uniquement de subsomption et de sous-types d'événements) et celle de 'partie-de', mais explicitement restreinte à la temporalité (c'est-à-dire que l'événement de la phrase élaborante doit être inclus temporellement dans celui de la phrase élaborée - ou, cas limite, équivalent à celui de la principale).

Le point nouveau, c'est l'abandon de la disjonction entre la condition de subsomption et celle de partie-de ou de sous-événement. La relation de subsomption devient la condition définitoire: 'in Elaboration, the evidence is that b is a subtype of a' (Asher et Lascarides, 2003: 206 et 461) et celle de 'partie-de' n'est plus qu'une conséquence temporelle de la première: ${ }^{26}$

Temporal Consequence of Elaboration:

\footnotetext{
${ }^{25}$ Cf. aussi Asher et Vieu (2005).

${ }^{26} \mathrm{Cf}$. 'Elaboration $(a, b)$ is a good "guess", so long as there's evidence that $b$ is a subtype of $a$ that is, there's evidence that $b$ 's eventuality is part of $a$ 's' (Asher et Lascarides, 2003: 204).
} 
Elaboration (a,b) $\longrightarrow$ Part-of (ea, eb) (Asher et Lascarides, 2003: 160)

Du coup, de nouvelles propriétés sont assignées à Élaboration comme la transitivité et la distributivité: ${ }^{27}$

\footnotetext{
'Since Elaboration implies that the main eventualities of its second argument are a mereological part of the main eventuality of its first argument, Elaboration is both transitive and distributes over segments' (Asher et Lascarides, 2003: 161).
}

Mais ce ne sont pas ces propriétés qui nous retiendront: la conséquence la plus importante pour nous est que l'hétérogénéité de la définition disjonctive d'Asher (1993) se trouve surmontée, puisque toute Élaboration est subsomptive et satisfait à la condition de 'partie-de' ou d'inclusion temporelle. Les phrases élaborantes dont l'événement recouvre celui de l'élaborée ne sont pas hors jeu, dans la mesure où le cas limite de l'inclusion est le recouvrement total des deux. Autre conséquence de poids: les emplois de 'partie-de' qui ne sont pas subsomptifs se trouvent écartés. Les exemples du type de indem de ci-dessus ne pourront ainsi plus être analysés comme des exemples d'Élaboration.

Notre analyse de la disjonction inclusive d'Asher a toutefois montré qu'il n'était guère possible d'avoir en même temps subsomption et relation 'partie-de': la même critique que nous avons émise contre la troisième possibilité de la disjonction inclusive d'Asher s'applique donc à la conception proposée dans Asher et Lascarides (2003). Et nous rejetons en conséquence l'idée que la subsomption d'Élaboration a pour suite temporelle une relation 'partie-de' ou d'inclusion temporelle de l'événement dénoté par $b$ dans l'événement dénoté par $a$, même si on intègre, tout logiquement, le cas de recouvrement. Nous rappellerons simplement que ce qui est à l'origine de cette erreur ce sont des exemples du type de:

(20) Max had a lovely meal last night. He ate lots of salmon.

où, apparemment, l'événement de la phrase élaborante est à la fois subsumé par l'événement de l'élaborée et en est une partie seulement.

Soulignons toutefois que si on renonce à voir dans subsomption une relation type $\longrightarrow$ sous-type, où l'implication unilatérale $(\mathrm{b} \longrightarrow \mathrm{a}$, mais a $\longrightarrow$ b) ne tient plus et si on entend simplement y voir, de façon plutôt lâche, le fait qu'un tout englobe en quelque sorte ses parties comme un type englobe ses sous-types, alors on peut maintenir la définition non disjonctive d'Asher et Lascarides et dire que l'Élaboration a comme contrainte la subsomption et comme conséquence temporelle l'inclusion de l'événement dénoté par l'élaborante dans celui dénoté par l'élaborée, étant donné que la possibilité pour la relation d'inclusion d'équivaloir à l'égalité permet,

27 Pour une explication de ces deux propriétés, voir Asher et Lascarides (2003). 
comme nous l'avons déjà souligné, d'intégrer les cas de subsomption où les deux événements sont identiques. Un avantage à cette logique plus molle: on récupère les emplois "partitifs » du type de l'escalade du Mont Cervin, puisque l'on pourra parler de subsomption à partir du moment où il $\mathrm{y}$ a une relation 'partie-de' temporelle entre les événements des deux phrases reliées. Mais ce faisant, il faut bien s'en rendre compte, on perd en finesse d'analyse, puisque la différence entre la relation hiérarchique de type 'hypo / hyperonymie' et la relation méréologique de type 'partie-tout' se trouve d'une certaine manière effacée, ce qui n'est pas souhaitable et ce qui aboutit d'ailleurs à rendre le concept de subsomption inutile, puisque l'indication de 'partie-de' pourra, en faisant jouer chaque fois qu'il le faut le cas de recouvrement total, intégrer tous les cas rencontrés.

\section{CONCLUSION}

La difficulté principale de la définition de la relation d'Elaboration vient du fait qu'elle implique crucialement le même et l'autre, puisque la phrase élaborante est intuitivement présentée comme ne faisant que préciser ou développer le contenu de la phrase élaborée. Une telle situation entraîne une tension entre les deux pôles opposés de l'identité et de la différence. Si l'on bascule trop du côté du même, on est conduit à tenir pour mineures les informations apportées par la phrase élaborante et surtout la porte est ouverte aux répétitions, paraphrases et autres reformulations. Si l'on essaie, par contre, de saisir en quoi consiste le « détail » ou l'information nouvelle apportée par la seconde phrase sur la première, alors nous guette le danger de l'éclatement de la relation en plusieurs sous-types de relations et on risque de mettre sur pied une catégorie hétérogène difficilement contrôlable.

Nous avons d'abord souligné que, déjà au niveau des approches intuitives, la tension entre identité et différence était bien présente et qu'elle se manifestait plus ou moins nettement dans les caractérisations et indices de reconnaissance proposés. Nous avons essayé ensuite de montrer qu'elle restait de mise dans les définitions formelles de la relation. Après avoir transité par la conception de Mann et Thompson (1988), nous avons analysé en détail la voie de l'inférence commune tracée par Hobbs (1979 et 1990), orientée vers l'identité, en essayant de mettre au jour les tenants et les aboutissants de la définition postulée, en indiquant notamment quels emplois elle pouvait traiter et lesquels lui échappaient. Nous avons abordé ensuite dans la même optique les approches formelles tournées vers la différence entre phrase élaborante et phrase élaborée d'Asher (1993) et d'Asher et Lascarides (2003): toutes les deux connaissent des difficultés sérieuses, parce que toutes les deux, quoique de façon un peu différente, postulent qu'il peut $\mathrm{y}$ avoir en même temps subsomption et relation méréologique. Au bout de notre parcours s'impose un résultat: celui de reprendre cette relation d'Élaboration dans le sens de la différenciation. 
Cela ne peut se faire qu'en travaillant sur des exemples variés (cf. les recherches sur corpus annotés, voir Prasad et al., 2007) avec des tests linguistiques, quasiment inexistants dans les études sur les relations de discours, permettant de mieux contrôler la pertinence linguistique des hypothèses définitoires avancées.

Address for correspondence:

Georges Kleiber \& Hélène Vassiliadou

Université de Strasbourg

UFR PLISE - Bât. Le Portique

Département Sciences du langage

14, rue René Descartes

BP 80010

$F$ - 67084 STRASBOURG Cedex

email: kleiber@unistra.fr \& vassili@unistra.fr

\section{REFERENCES}

Asher, N. (1993). Reference to Abstract Objects. Dordrecht: Kluwer.

Asher, N. and Lascarides, A. (1996). Lexical disambiguation in a discourse context. In: J. Pustejovsky and B. Boguraev (eds.), Lexical Semantics: the problem of polysemy. Oxford: Clarendon Press, pp. 69-108.

Asher, N. and Lascarides, A. (2003). Logics of Conversation. Cambridge: Cambridge University Press.

Asher, N. and Vieu, L. (2005). Subordinating and coordinating discourse relations. Lingua 115: 591-610.

Behrens, B. (1998). Contrastive Discourse: An interlingual approach to the interpretation and translation of free ING-participial adjuncts. Ph.D. thesis, University of Oslo.

Busquets, J., Vieu, L. et Asher, N. (2001). La SDRT: une approche de la cohérence du discours dans la tradition de la sémantique dynamique. Verbum, XXIII(1): 73-101.

Cornish, F. (1999). Anaphora, Discourse, and Understanding. Evidence from English and French. Oxford: Oxford University Press.

Cornish, F. (2006a). Relations de cohérence en discours: critères de reconnaissance, caractérisation et articulation cohésion-cohérence. Corela, (revue en ligne) $n^{\circ}$ spécial: 1-22.

Cornish, F. (2006b). Relations de cohérence et anaphores en contexte interphrastique: une symbiose parfaite. Langages, 163: 37-55.

Cornish, F. (à par.). Inter-sentential anaphora and coherence relations in discourse: A perfect match. Language Sciences. 
Degand L. (1998). On classifying connectives and coherence relations. In: M. Stede, L. Wanner and E. Hovy (eds.), Proceedings of the ACL Workshop on Discourse Relations and Discourse Markers, pp. 29-35.

Eggins, S. (1994). An Introduction to Systemic Functional Linguistics. London / New York: Continuum.

Fabricius-Hansen, C. and Behrens, B. (2001). Elaboration and related discourse relations viewed from an interlingual perspective. Sprik_Report $13: 1-34$.

Gernsbacher, M. and Givón, T. (eds.) (1995). Coherence in Spontaneous Text. Amsterdam: John Benjamins.

Grabski, M. (2000). Satztopik und Diskurstopik in Elaboration-Kontexten. In: K. Schwabe et al., Issues on Topics, ZAS Papers in Linguistics, 20: 173-207.

Grosz, B. and Sidner, C. (1986). Attention, intentions, and their structure of discourse. Computational Linguistics, 12-3: 175-203.

Halliday, M.A.K. (1994). An Introduction to Functional Grammar. London: Edward Arnold.

Hobbs, J.R. (1979). Coherence and Coreference. Cognitive Science, 3: 6790.

Hobbs, J.R. (1990). The coherence and structure of discourse. In: Literature and Cognition. Leland Stanford Junior University, Calif.: CSLI Lecture Notes, pp. 83-114.

Hume, D. (1955, 1748). A Treatise of Human Nature. Oxford: Clarendon Press, 3 vols.

Kamp, H. and Rohrer, C. (1983). Tense in texts. In: R. Bäuerle, C. Schwarze and A. von Stechov (eds.), Meaning, Use and Interpretation of Language, Berlin / New York: Walter de Gruyter, pp. 251-269.

Kehler, A. (2004). Discourse Coherence. In: L.R. Horn and G. Ward (eds.), The Handbook of Pragmatics, Oxford: Blackwell Publishing, pp. 241-265.

Kleiber, G. et Vassiliadou, H. (2007). Sur les approches intuitives de la relation d'Elaboration. Scolia, 22: 147-161.

Knott, A. (2001). Semantic and pragmatic relations and their intended effects. In: T. Sanders, J. Schilperoord and W. Spooren (eds.), Text Representation: Linguistic and Psycholinguistic Aspects. Amsterdam: John Benjamins, pp. 127-152.

Knott, A. and Dale, R. (1994). Using linguistic phenomena to motivate a set of coherence relations. Discourse Processes, 18(1): 35-62.

Knott, A. and Sanders, T. (1998). The classification of coherence relations and their linguistic markers: an exploration of two languages. Journal of Pragmatics, 30: 135-175.

Knott, A., Oberlander, J., O’Donnell, M. and Mellish, C. (2001). Beyond elaboration: the interaction of relations and focus in coherent text. In: $\mathrm{T}$. Sanders, J. Schilperoord and W. Spooren (eds), Text Representation: 
Linguistic and Psycholinguistic Aspects. Amsterdam: John Benjamins, pp. 181-196.

Mann, W.C. and Thompson, S. (1987). Rhetorical Structural Theory: A Theory of text organization. Los Angeles: University of Southern California.

Mann, W.C. and Thompson, S. (1988). Rhetorical structure theory: toward a functional theory of text organization. Text, 8: 243-281.

Mannn, W.C. and Taboada, M. (2005). Rhetorical Structure Theory: Relation definitions. Site Internet www.sfu.ca/rst/01intro/definitions.html, 1-14.

Martin, J. (1992). English Text: System and Structure. Amsterdam: John Benjamins.

Oates, S.L. (1999). State of the Art Report on Discourse Markers and Relations. ITRI-99-08, University of Brighton.

Prasad, R., Miltsakaki, E., Dinesh, N., Lee, A., Joshi, A., Robaldo, L. and Webber, B-L. (2007). The Penn Discourse TreeBank 2.0. Annotation Manual. Technical report, IRCS, Institute for Research in Cognitive Science, University of Pennsylvania.

Rossari, C. (2000). Connecteurs et relations de discours: des liens entre cognition et signification. Nancy: Presses Universitaires de Nancy.

Rossari, C. (2001). Les relations de discours: approches rhétoriques, approches pragmatiques et approches sémantiques. Verbum, XXIII (1): 59-72.

Sanders, T. (1997). Semantic and pragmatic sources of coherence: on the categorisation of coherence relations in context. Discourse Processes, 24: 119-147.

Sanders, T., Spooren, W. and Noordmann, L. (1992). Toward a taxonomy of coherence relations. Discourse Processes, 15: 1-35.

Sanders, T., Spooren, W. and Noordmann, L. (1993). Coherence relations in a cognitive theory of discourse representation. Cognitive Linguistics 4: 93133.

Scott, D.R. and de Souza, C.S. (1990). Getting the message across in RSTbased text generation. In: Dale, R., Mellish, C. and Zock, M. (eds.), Current Research in Natural Language Generation, London: Academic Press, pp. 47-73.

Taboada, M. and Mann, W.C. (2006). Rhetorical Structure Theory: Looking back and moving ahead. Discourse Studies, 8-3: 423-459. 